\title{
Representation and Calibration of Elastic Localization Kernels for a Broad Class of Cubic Polycrystals
}

\author{
Yuksel C. Yabansu ${ }^{1}$ and Surya R. Kalidindi ${ }^{1,2^{*}}$ \\ ${ }^{1}$ George W. Woodruff School of Mechanical Engineering, Georgia Institute of Technology, Atlanta, GA \\ 30332, USA \\ ${ }^{2}$ College of Computing, Georgia Institute of Technology, Atlanta, GA 30332, USA \\ *corresponding author
}

\begin{abstract}
Localization kernels play an important role in the study of hierarchical material systems with well separated length scales. They allow for a computationally efficient communication of critical information between the constituent length scales. They are particularly well suited for capturing how an imposed variable (e.g., stress or strain) at the higher length scale is spatially distributed at the lower length scale (i.e., localization linkages). In recent work, our research group has presented a novel framework called Materials Knowledge Systems (MKS) for the representation and calibration of the localization kernels, and demonstrated the viability of this approach on selected individual material systems. In this work, we present and demonstrate an important extension to the MKS framework that allows representation and calibration of the localization kernels for an entire class of materials (e.g., a selected class of single phase cubic polycrystalline materials).
\end{abstract}

Keywords: Materials Knowledge Systems; Localization kernels; Generalized spherical harmonics; Legendre polynomials; Hierarchical multiscale modeling 


\section{Introduction}

Most advanced material systems of interest to emerging technologies exhibit rich hierarchical internal structures with well separated length scales. The mechanical response of such material systems has been addressed rigorously in prior literature using generalized composite theories [112]. Inherent to these theories is the concept of a scale-bridging localization tensor that relates the local fields of interest at the microscale to the macroscale (typically averaged) fields. For example, the fourth-rank localization tensor for elastic deformation, $\boldsymbol{a}$, can be defined to relate the local elastic strain at any location of interest in the microstructure to the macroscale strain imposed on the composite material system as:

$\boldsymbol{\varepsilon}(x)=\boldsymbol{a}(x)\langle\boldsymbol{\varepsilon}(x)\rangle$

$\boldsymbol{a}(x)=\left(\boldsymbol{I}-\left\langle\boldsymbol{\Gamma}\left(x, x^{\prime}\right) \boldsymbol{C}^{\prime}\left(x^{\prime}\right)\right\rangle+\left\langle\boldsymbol{\Gamma}\left(x, x^{\prime}\right) \boldsymbol{C}^{\prime}\left(x^{\prime}\right) \boldsymbol{\Gamma}\left(x^{\prime}, x^{\prime \prime}\right) \boldsymbol{C}^{\prime}\left(x^{\prime \prime}\right)\right\rangle-\cdots\right)$

In Eq. (2), $\boldsymbol{I}$ is the fourth-rank identity tensor, $\boldsymbol{C}^{\prime}(x)$ is the deviation in the local elastic stiffness at spatial location $\mathbf{x}$ with respect to that of a selected reference medium, $\boldsymbol{\Gamma}$ is a symmetrized derivative of the Green's function defined using the elastic properties of a selected reference medium $[1,10,13]$, and \langle\rangle brackets denote an ensemble average over a representative volume element (RVE) of the material microstructure.

Equation (2) can be transformed into a more computationally useful form by taking advantage of the concept of spatially resolved microstructure function $m(x, n)$ [14] that reflects the probability density associated with finding the local state $n$ (to within an invariant measure $d n)$ at the spatial location $x$ (note that $m(x, n) d n$ reflects the corresponding probability). The local state identifies the specific combination of local features (including phase identifiers, elemental compositions, crystal lattice orientations, etc.) needed to uniquely define the relevant local physical properties at the spatial location $x$. Furthermore, the complete set of all distinct 
local states that are possible in a given material system is referred to as the local state space, denoted by $H$ (i.e., $n \in H$ ). Introducing this concept, invoking the ergodic hypothesis, and substituting $r=x-x^{\prime}$, one can recast Eq. (1) as $[15,16]$ :

$$
\begin{aligned}
& \boldsymbol{\varepsilon}(x)=\left(\boldsymbol{I}-\int_{R} \int_{H} \boldsymbol{\alpha}(r, n) m(x+r, n) d n d r\right. \\
& \left.\quad+\int_{R} \int_{R} \int_{H} \int_{H} \widetilde{\boldsymbol{\alpha}}\left(r, r^{\prime}, n, n^{\prime}\right) m(x+r, n) m\left(x+r+r^{\prime}, n^{\prime}\right) d n d n^{\prime} d r d r^{\prime}-\cdots\right)\langle\boldsymbol{\varepsilon}(x)\rangle
\end{aligned}
$$

The structure of Eq. (3) offers many computational advantages. First, the terms $\boldsymbol{\alpha}(r, n)$ and $\widetilde{\boldsymbol{\alpha}}\left(r, r^{\prime}, n, n^{\prime}\right)$ are independent of the microstructure function. In other words, they capture the microstructure-independent physics governing the local elastic response of a composite material. Second, the terms in Eq. (3) can be efficiently computed using discrete Fourier transforms (DFTs). Consequently, the terms $\boldsymbol{\alpha}(r, n)$ and $\widetilde{\boldsymbol{\alpha}}\left(r, r^{\prime}, n, n^{\prime}\right)$ are referred to as first-order and second-order localization kernels (or influence functions), respectively. Note that Eq. (3) represents an infinite series expansion of a highly nonlinear function, where each term of the series captures a linearized contribution from a specific topological feature in the microstructure.

There have been essentially two main difficulties in the computation of the localization kernels defined in Eq. (3). The first difficulty stems from the fact that $\boldsymbol{\Gamma}(r)$ (embedded in the localization kernels; see Eq. (2)) exhibits a singularity at $r=0$. The second difficulty is that the convergence of the series is quite sensitive to the selection of the reference medium (e.g., [17]). In an effort to overcome these impediments, our research group has developed a novel data-driven framework called Materials Knowledge System (MKS) [18-24]. In the MKS approach, localization kernels are obtained by a calibration procedure that involves matching the predictions of Eq. (3) to the corresponding predictions from previously validated numerical models (e.g., finite element models) for a broad range of exemplar microstructures. The central advantage of the MKS 
methodology lies in its computational efficiency. Once the localization kernels are calibrated, they can be applied to new microstructures with very little computational cost, often orders of magnitude lower than what is needed to execute the previously established numerical model. The viability and the computational advantages of the MKS approach have been successfully demonstrated for thermo-elastic deformation fields in composites [19], rigid-viscoplastic deformation fields in composites [18], the evolution of the composition fields in spinodal decomposition of binary alloys [21], and the elastic deformation fields in single-phase polycrystalline aggregates [24].

It is noted here that all of the prior case studies in MKS have utilized a single descriptor of the local state (usually the phase identifier or the crystal lattice orientation or the local chemical composition). It is anticipated that most advanced materials explored in emerging technologies will demand the use of complex descriptors for the local state. In this paper, we present a generalized framework that allows the combined use of multiple descriptors for the local state. More specifically, building on our earlier work [9, 24-30], we demonstrate the tremendous advantages of Fourier representations of the localization kernels in arriving at compact representations that facilitate easy calibration over extremely large domains of interest (covering a very broad range of material systems). Since the calibration process needs to be performed only once, the extended MKS framework presented here opens a completely new and practical approach for addressing multiscale hierarchical modeling and simulations [31-39]. More importantly, the extensible approach presented here allows community-wide sharing and curation of the core materials knowledge through the potential establishment of an e-library of localization kernels. This is mainly facilitated by the fact that the localization kernels in the MKS framework are designed to be independent of the microstructure function. 


\section{The Generalized MKS Framework}

We seek a computationally efficient form of Eq. (3) using spectral representations. Specifically, we seek representations of the following type for the various functions in Eq. (3) (only the functions in the first term are shown below; the representations can be extended in future to higher-order terms in the series):

$m(x, n)=\sum_{L} \sum_{S} M_{S}^{L} Q_{L}(n) \chi_{S}(x), \quad \boldsymbol{\alpha}(r, n)=\sum_{L} \sum_{t} \boldsymbol{A}_{t}^{L}(n) Q_{L}(n) \chi_{t}(r)$

In Eq. (4), $Q_{L}(n)$ is a suitably selected Fourier basis for functions defined on the local state space (examples will be provided later) with the following orthonormal properties:

$\int_{H} Q_{L}(n) Q_{L^{\prime}}^{*}(n) d n=\frac{\delta_{L L^{\prime}}}{N_{L}}$

where the superscript * denotes a complex conjugate, $\delta_{L L^{\prime}}$ is the Kronecker delta, and $N_{L}$ is a constant that might depend on $L . \chi_{S}(x)$ in Eq. (4) defines an indicator basis which essentially tessellates the spatial domain into a uniform grid [14]. This function is defined such that its value is one for all points belonging to spatial bin $s$, and zero for all points outside. The choice of the indicator basis for the spatial variables in Eq. (4) is primarily motivated by the fact that it allows for the use of discrete Fourier transforms (DFTs) in carrying out the integrals in Eq. (3). Using the orthogonal properties of both bases, we can show

$M_{s}^{L}=\frac{N_{L}}{\Delta} \int_{H, V} m(x, n) Q_{L}^{*}(n) \chi_{s}(x) d n d x \quad \boldsymbol{A}_{t}^{L}=\frac{N_{L}}{\Delta} \int_{H, R} \boldsymbol{\alpha}(r, n) Q_{L}^{*}(n) \chi_{t}(r) d n d r$

where $\Delta$ is the volume of the spatial bin.

Introducing these spectral representations into Eq. (3), we derive here a generalized form of the MKS: 
$\boldsymbol{p}_{s}=\left(\sum_{L} \sum_{t} \frac{\Delta}{N_{L}} \boldsymbol{A}_{t}^{L} M_{s+t}^{L}+\sum_{L} \sum_{L^{\prime}} \sum_{t} \sum_{t^{\prime}} \frac{\Delta^{2}}{N_{L} N_{L^{\prime}}} \boldsymbol{A}_{t}^{L L^{\prime}} M_{s+t}^{L} M_{s+t+t^{\prime}}^{L^{\prime}}+\cdots\right)\langle\boldsymbol{p}\rangle$

It is pointed that if the $Q_{L}(n)$ were selected to be the indicator functions (i.e., a simple binning of the local state space), we would recover the simpler MKS formulation utilized in our prior studies involving multiphase composites [18-20, 22, 39]. Likewise, if the local state was selected to the crystal lattice orientation and the Fourier basis was selected to the generalized spherical harmonics (GSHs) [40], we would recover the MKS formulation we have recently demonstrated for elastic deformations in single phase polycrystalline microstructures [24]. We believe that the formulation presented above is the most general and practical MKS formulation that will be applicable for a very broad range of advanced material systems.

\section{[Insert Figure 1 here]}

The similarity of the generalized MKS formulation presented here to the versions in our prior work [19-23] suggests the use of the same overall strategy (from our prior work) for calibrating the influence coefficients (such as $\boldsymbol{A}_{t}^{L}$ ) in Eq. (7). The overall workflow involved in building the MKS databases is shown in Figure 1 as a broadly usable template. This procedure involves four different main tasks (color coded in Figure 1) with several subtasks. Although this template has not been presented before, the protocols described in all of our prior MKS case studies essentially followed these protocols.

\section{Case Study: Application to Elastic Deformations in a Family of Cubic Polycrystals}

In all prior applications of the MKS approach, one material system was considered at a time. In other words, the developed MKS databases were applicable only to a broad range of microstructures within a selected material system. In this study, we demonstrate the viability of the generalized MKS framework presented in this paper to establish MKS databases to elastic deformations in an entire family of cubic polycrystals. If this approach becomes viable, then it 
would open up new avenues for collaborative research where such broadly applicable databases are generated and shared in open-access and open-source repositories to allow community driven curation and adoption in multiscale materials modeling and simulation efforts [41].

In this case study, we focus on the application of the generalized MKS framework introduced in the previous section to a family of cubic polycrystals. For this class of microstructures, the local state description needs to include both the crystal lattice orientation and the single crystal cubic elastic stiffness constants (expressed in the crystal reference frame). The crystal lattice orientation can be expressed by a set of ordered Bunge-Euler angles, $g=\left(\varphi_{1}, \Phi, \varphi_{2}\right)$. In prior work $[24,26]$, we have shown that functions defined on the orientations space can be efficiently represented using symmetrized GSH functions, $\dot{T}_{l}^{\mu \nu}$, as a Fourier basis. More specifically, it was demonstrated that the elastic localization kernels for single phase cubic material needed only 10 terms in these GSH representations.

In this paper, we seek to establish localization relationships for an entire family of single phase cubic polycrystals (as opposed to doing this for each material system at a time). In order to accomplish this ambitious task, it is necessary to include the fundamental cubic single crystal elastic stiffness constants, $\left(C_{11}, C_{12}, C_{44}\right)$, in the local state description. In other words, the complete local state description for this problem can be expressed as $n=\left(g, C_{11}, C_{12}, C_{44}\right)$. Note that this local state description now has six independent variables (recall that $g=\left(\varphi_{1}, \Phi, \varphi_{2}\right)$ ). This compounded local state description is needed to accurately represent the fourth-rank elasticity tensors in the sample reference frame in each spatial bin of the microstructure of interest (which now can correspond to a broad range of cubic polycrystals).

The next task is the selection of the Fourier basis, $Q_{L}(n)$ (see Eq. (4)). While we already know that $\dot{T}_{l}^{\mu \nu}$ can serve as an excellent basis for the functional dependence on crystal lattice 
orientation, it is not clear what basis should be used for the other variables $\left(C_{11}, C_{12}, C_{44}\right)$. The main challenge here arises from the fact that these variables are not defined on a periodic domain (unlike $g$ ). In other problems of this ilk [42], our experience has taught us that Legendre polynomials $P(x)$ can be used as a Fourier basis in these situations. In order to employ these basis functions, we need to rescale the variables such that their domain is mapped to the interval $(-1,1)$. This can be accomplished generically for any variable $C$ by defining a new variable

$C^{r}=\frac{2 C-C_{\min }-C_{\max }}{C_{\max }-C_{\min }}$

where $C_{\min }$ and $C_{\max }$ are the respective minimum and maximum values of $C$. Applying Eq. (8) to $\left(C_{11}, C_{12}, C_{44}\right)$ results in rescaled descriptors $\left(C_{11}^{r}, C_{12}^{r}, C_{44}^{r}\right)$.

In summary, we might now describe the extended local state for the material class of interest as $n=\left(g, C_{11}^{r}, C_{12}^{r}, C_{44}^{r}\right)$ with the Fourier basis selected as

$Q_{L}(n)=Q_{L}\left(g, C_{11}^{r}, C_{12}^{r}, C_{44}^{r}\right)=\dot{T}_{l}^{\mu \nu}(g) P_{a}\left(C_{11}^{r}\right) P_{b}\left(C_{12}^{r}\right) P_{c}\left(C_{44}^{r}\right)$

In Eq. (9), $L$ enumerates each distinct combination of $(\mu, v, l, a, b, c)$ and the normalization factor, $N_{L}$, can be expressed as

$N_{L}=\frac{8}{(2 l+1)(2 a+1)(2 b+1)(2 c+1)}$

Next, we describe the procedures used to build the localization linkages using the template shown in Figure 1.

\subsection{Generation of calibration data set}

The generation of calibration data set (green color coded section in Figure 1) starts with the identification of the local state space corresponding to the selected local state descriptors. Since $n=\left(g, C_{11}^{r}, C_{12}^{r}, C_{44}^{r}\right)$ the overall local state space is essentially the compounded local state spaces 
of all the variables involved. The local state space for the crystal lattice orientation is simply the cubic-triclinic fundamental zone, $F Z_{C}$, defined as

$$
\begin{gathered}
F Z_{C}=\left\{g=\left(\varphi_{1}, \Phi, \varphi_{2}\right) \mid 0 \leq \varphi_{1} \leq 2 \pi, \cos ^{-1}\left(\frac{\cos \varphi_{2}}{1+\cos ^{2} \varphi_{2}}\right) \leq \Phi \leq \frac{\pi}{2},\right. \\
\left.0 \leq \varphi_{2} \leq \frac{\pi}{4}\right\}
\end{gathered}
$$

This essentially means that each spatial bin of the microstructure can be assigned any combination of crystal lattice orientations from $F Z_{C}$. Although the framework presented above allows assignment of polycrystals at each spatial bin, we will restrict our attention here to the assignment of only single crystals in each spatial bin. In other words, we will assume that the spatial discretization used is such that only one crystal orientation is assigned in each spatial bin. The next logical question is whether or not we need to use all of the orientations in $F Z_{C}$ in the calibration step. In prior work, we have demonstrated that the complete set of all theoretically possible orientation distributions forms a texture hull in the GSH space $[9,26,29,43]$. The vertices of the texture hull correspond to single crystal textures (i.e., the orientation distributions are essentially delta functions). The set of crystal lattice orientations corresponding to the vertices of the texture hull have been called principal orientations [9]. Our hypothesis is that it is adequate to use the principal orientations in the calibration step. The reason for this is that the single crystal orientation distribution for any of the other orientations would constitute an interpolation of the single crystal orientation distributions for the principal orientations. However, the identification of the principal orientations is not a trivial task especially when one has to deal with a large number of Fourier dimensions.

For cubic-triclinic symmetry of interest in the present case study, the spectral representation of the elastic stiffness tensor in the orientation space is expected to include all $l \leq 4 \mathrm{GSH}$ terms 
[24]. Taking into account the fact that GSH coefficients for real functions have certain interdependencies (some of the coefficients will be complex conjugates of the others), the GSH representations for $l \leq 4$ constitute 10 dimensions. However, the visualization of cubic-triclinic texture hull in 10 dimensions in order to identify the principal orientations is a major challenge. In this paper, we utilized principal component analysis (PCA) to address this challenge. PCA essentially provides a linear transformation of high dimensional data into a new orthonormal coordinate frame, whose axes are ordered by the amount of the variance observed in the dataset (with the first axis identifying the direction of maximum variance in the dataset). The same idea was used in prior work in a different context $[44,45]$. Figure 2 shows PCA representations of the single crystal orientation distributions corresponding to orientations sampled from $F Z_{C}$. The sample orientations are color-coded such that the orientations on the surface of $F Z_{C}$ are marked red, while the orientations inside $F Z_{C}$ are marked blue. It can be seen from the PCA plots (low dimensional projections) in Figure 2 that the orientations on the surface of $F Z_{C}$ are indeed the principal orientations of interest. Based on this observation, 220 principal orientations are selected from the bounding surface of $F Z_{C}$ for the calibration step in this study.

\section{[Insert Figure 2 here]}

Next we decide on the ranges for the elastic stiffness constants $\left(C_{11}, C_{12}, C_{44}\right)$. The ranges were selected to include many of the commonly used cubic metals, while covering cubic anisotropy ratios (defined as $Z=2 C_{44} /\left(C_{11}-C_{12}\right)$ [9]) in the range $Z=[0.5,1.95]$. This range is expected to cover the range of low to moderate contrast for which the first term in the MKS series is deemed adequate based on our prior experience [18-21, 24]. It is also pointed out that $Z=1$ corresponds to the isotropic case. Because of the rescaling described earlier (see Eq. (8)), the range for the rescaled local state variables will be $(-1,1)$ independent of the selection of the ranges for 
$\left(C_{11}, C_{12}, C_{44}\right)$. Within the selected range, a total of 64 distinct sets of $\left(C_{11}, C_{12}, C_{44}\right)$ were used in generating the calibration dataset in this work. These sets are identified in Figure 3.

[Insert Figure 3 here]

Once the local state and local state space are identified, next steps in generating the calibration data set are the selection of the classes of representative microstructures of interest (steps 2 and 3). The selection of microstructures used in the calibration data set plays a pivotal role in the accuracy of the MKS linkage established. Following the strategies described in prior work [24], we have decided to use a total of three classes of microstructures in the calibration step in the present case study: delta, equiaxed, and random microstructures (see Figure 4). A delta microstructure consists of one spatial bin (or voxel) of one crystal orientation in the center of MVE (microstructure volume element) surrounded completely by spatial bins of a different crystal orientation. The equiaxed microstructures are generated from three dimensional Voronoi cells formed around points randomly placed in the MVE, where each cell is assigned a distinct crystal lattice orientation. The random microstructures are produced simply by randomly assigning the crystal lattice orientation to each spatial bin of the MVE.

\section{[Insert Figure 4 here]}

The final step in generating the calibration data set is to execute the micromechanical finite element (FE) simulations using suitable boundary conditions (steps 4 and 5). Periodic boundary conditions were imposed on all MVEs used in the calibration in such a way that all macroscopic strain components other than $\varepsilon_{11}$ had zero values. Repeating the simulations with six different sets of boundary conditions allows us to establish the MKS linkages for all components of the fourth rank elastic localization kernels [16, 20,39]. In the present report, we limit our attention to only the $\varepsilon_{11}$ strain component. A uniformly discretized grid of $21 \times 21 \times 21$ is used for each 
MVE. Each spatial bin in the MVE is defined as an 8-noded, three-dimensional, solid element (C3D8) in the finite element mesh. A total of 1800 microstructures (600 of each of the three types of microstructures shown in Figure 4) compounded with 64 different assignments of single crystal elastic constants (shown in Figure 3) produced the calibration dataset used in this work. The finite element simulations were carried out using the commercial software ABAQUS [46].

\subsection{Calibration of influence coefficients}

The next major task in the MKS workflow is the estimation of influence coefficients (blue color coded section in Figure 1). As mentioned earlier, in the MKS approach, influence coefficients are calibrated to micromechanical finite element simulations through the application of linear regression methods in the DFT space. In this paper, the localization relationships will be limited to the first term in Eq. (7). In our previous studies [18-20, 24], it was shown that the first term captures adequately the MKS linkages for material systems with low to moderate contrast in the properties of microscale constituents (as is the case in the example selected here).

The first step in the calibration of influence coefficients is the spectral representation of each of the MVE included in the calibration set. Let $\left(g_{s}, C_{11 s}^{r}, C_{12 s}^{r}, C_{44 s}^{r}\right)$ denote the local state in the spatial bin $s$. The spectral representation of the microstructure function can then be written as

$M_{s}^{L}=\frac{N_{L}}{\Delta} \dot{T}_{l}^{\mu \nu *}\left(g_{s}\right) P_{a}\left(C_{11 s}^{r}\right) P_{b}\left(C_{12 s}^{r}\right) P_{c}\left(C_{44 s}^{r}\right)$

Next, the microstructure function and the finite element predicted response field of each MVE are transformed into DFT space. As shown in our prior work [18-21, 24], this step effectively decouples the influence coefficients, making the calibration a much simpler task. This benefit is a result of the well-known convolution properties of the DFTs. Making use of these properties, the first term of the localization relationships in Eq. (7) is expressed as 


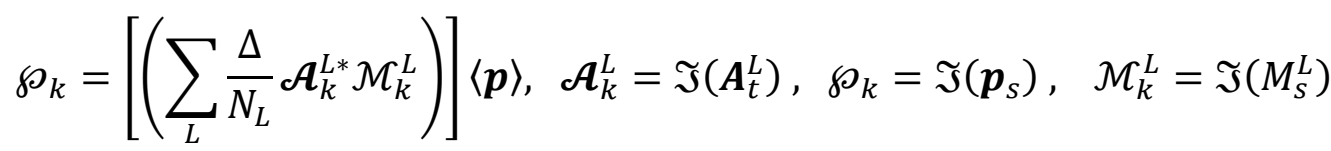

where $\mathfrak{J}($ ) denotes the DFT operation with respect to the spatial variables $s$ or $t$. The decoupling in Eq. (13) (notice that there is no summation over $k$ ) allows a relatively simple calibration of $\mathcal{A}_{k}^{L}$ using ordinary least squares (OLS) method. The OLS formulation of Eq. (13) can be expressed as

$\sum_{r} \omega \wp_{k}\left(\omega^{\mathcal{M}} \mathcal{M}_{k}^{L^{\prime}}\right)^{*}=\sum_{L} \frac{\Delta}{N_{L}} \mathcal{A}_{k}^{L *}\left[\sum_{\omega} \omega^{\mathcal{M}} \mathcal{M}_{k}^{L}\left({ }_{\omega} \mathcal{M}_{k}^{L^{\prime}}\right)^{*}\right]\langle\boldsymbol{p}\rangle$

where $\omega$ enumerates over the calibration datasets. In Eq. (7), the total number of Fourier coefficients of influence functions, $\boldsymbol{A}_{t}^{L}$, is $\mathcal{L} \times S$, where $S$ is the total number of spatial cells in each MVE, and $\mathcal{L}$ is the number of basis functions used in the Fourier expansions of the microstructure function and the MKS kernel functions (i.e., the number of distinct values of $L$ in Eqs. (4), (7) and (9)). If OLS is performed directly on $A_{t}^{L}$ using Eq. (7), one needs to invert a matrix of size $(\mathcal{L S}) \times(\mathcal{L} S)$. The central advantage of transforming to the DFT space is that $\boldsymbol{A}_{k}^{L}$ can be estimated by performing OLS as shown in Eq. (14), which requires the inversion of a matrix of size $\mathcal{L} \times \mathcal{L}$. This operation, however needs to be performed $S$ times. Given that $S$ is typically much larger than $\mathcal{L}$, the strategy described in Eq. (14) represents a major computational advantage.

As the next task, we need to establish how many terms of the Fourier series we wish to retain (i.e., establish $\mathcal{L}$ ). Based on our past experience with similar problems [15, 16, 47], we anticipate that we will only need ten of the GSH basis functions (corresponding to $l=(0,4)$ ) for accurately representing the elastic MKS kernel functions for cubic polycrystals. Since we do not have any prior expectations on how many Legendre basis functions are needed to capture the functional 
dependence on the elastic stiffness parameters, we will have to execute a few trials to establish this number. Using the calibration dataset produced in Section 3.1, OLS was performed using Eq. (14) for different truncation levels on the Legendre basis, and the average error in each MVE of the calibration set was computed as

$E_{r}=\sum_{S=1}^{S} \frac{\left|p_{s}^{F E M}-p_{s}^{M K S}\right|}{S \times\langle p\rangle} \times 100$

where $p_{S}^{F E M}$ and $p_{S}^{M K S}$ are the response fields of interest in the spatial cell $s$ from MKS and FEM predictions, respectively. In this case study, it was decided to keep the truncation level the same for all three Legendre representations (one for each elastic stiffness parameter). Let $\mathcal{B}$ denote the number of terms retained in the Legendre series (same for all three elastic stiffness parameters). The value of $\mathcal{B}$ was increased systematically and the computed error using Eq. (15) is plotted in Figure 5. It was observed that truncation beyond $\mathcal{B}=3$ did not improve the accuracy of the linkage significantly. It is also worth noting that the average error values for $\mathcal{B}=3$ were extremely low $(<<1 \%)$. This result implies that the elastic MKS influence functions can be captured with adequate precision for the family of cubic polycrystals studied here with only $\mathcal{L}=270$ (i.e., 10 GSH terms and 3 terms for each Legendre basis) spectral coefficients. This is a remarkable compaction of an extremely complex physical phenomenon over a broad class of material systems. [Insert Figure 5 here]

\subsection{Generation of validation data set}

In order to evaluate the performance of the MKS linkages, a validation data (distinct from the calibration dataset produced in Section 3.1) was generated following the steps in red color coded section of Figure 1. For sampling of the local state space (step 1), MVEs of the validation set were assigned cubic single crystal elastic stiffness constants from the region shown in Figure 3 
that are distinct from the 64 combinations used in the calibration set. For crystal lattice orientations, the entire cubic-triclinic fundamental zone is utilized in generation of the validation set as opposed to the selection of orientations only from the boundary of fundamental zone used in the calibration. As a result of these protocols, the microstructures used in the validation are completely distinct from those used in the calibration. In steps 2 and 3, a validation set of 9000 microstructures comprising 3000 delta, 3000 equiaxed, and 3000 random microstructures with assignments of single crystal elastic stiffness constants as described above, were utilized. The $\varepsilon_{11}$ strain field for the validation dataset was predicted by executing FE simulations by applying the same boundary conditions used for calibration data set.

\subsection{Validation of $M K S$}

The last step of the MKS protocols is the validation of the MKS influence coefficients (purple color coded section in Figure 1). This can be achieved simply by comparing directly the predictions from the MKS linkages established in Section 3.2 with the validation dataset assembled in Section 3.3. The MKS predictions for the validation dataset are generated by using Eq. (13) directly. The extremely low computational cost of DFTs makes this a trivial task.

The accuracy and the robustness of the MKS linkages established in this work is shown in Figure 6 by plotting the mean, minimum, and maximum errors (defined in Eq. (15)) for different cubic anisotropy ratios used in both the calibration and validation datasets. As expected, the average error increases with higher levels of anisotropy (results in higher contrast levels in the composite material system). It is also shown that the MKS kernels established in this study are highly accurate (note that highest average error is well below 1\%).

\section{[Insert Figure 6 here]}


Figure 6 attests to the high accuracy of the MKS linkages established in the present case study based on the average error definition from Eq. (15). Next, we critically evaluate the predictions of the MKS linkages at the level of individual spatial bins. In Figure 7, the strain field predicted by the MKS approach for a selected equiaxed microstructure with an elastic anisotropy ratio of $Z=1.32$ is compared with the corresponding prediction from the $\mathrm{FE}$ simulation. Equiaxed microstructure shown in Figure 7(a) contained 157 distinct orientations randomly picked from the complete cubic-triclinic FZ. The middle slices of strain fields from MKS and FEM predictions are shown in Figure 7(c) and 7(d), respectively. Figure 7(b) shows the frequency plot of the strain distribution of entire MVE for both MKS and FEM predictions. From Figure 7, it can be seen that MKS and FEM predictions are almost indistinguishable from each other. It is noted that the average error for this example microstructure shown was only $0.11 \%$.

\section{[Insert Figure 7 here]}

For cubic polycrystals with higher levels of anisotropy corresponding to $Z=0.54$ and $Z=1.94$, the MKS predictions are compared with the corresponding FEM results in Figure 8. Even though the values of the average error have increased somewhat $(0.62 \%$ for the case with $Z=1.94$, and $0.59 \%$ for the case with $Z=0.54$ ), the accuracy of the MKS linkages established in this work is remarkable. This is particularly impressive when one keeps in mind the tremendous compaction achieved in the description of the influence functions over extremely large continuous local state spaces.

Other than the accuracy of MKS, the computational efficiency of MKS should also be emphasized. The FEM prediction of the microstructure in Figure 7(a) took 24 seconds with 2 
processors (each $3.0 \mathrm{GHz}$ ) on a supercomputer, while prediction with MKS took only 2 seconds with only 1 processor $(3.0 \mathrm{GHz})$ on a standard desktop computer.

\section{[Insert Figure 8 here]}

The influence functions are analogous to Green's functions and show a decaying characteristic with increasing size of $t$ vector. It was previously shown that the influence functions obtained from smaller domains can be extended to larger domains by padding with zeros $[20-22,24]$. This is done to facilitate the applications of the MKS kernels to arbitrary-sized MVEs (or RVEs) without much additional work (i.e., without the need to recalibrate the MKS linkages). In an effort to demonstrate the scalability of MKS framework, the Fourier coefficients of the influence functions obtained from the $21 \times 21 \times 21$ domain are extended to the larger domain of $45 \times 45 \times 45$ by zero-padding. For validation of the scalability of MKS for cubic polycrystalline aggregates, a $45 \times 45 \times 45$ equiaxed microstructure with 1257 distinct orientations from the entire cubic-triclinic FZ was generated.

Assigning a set of cubic elastic stiffness parameters with $Z=1.32$ to the microstructure, the FEM and MKS predictions were obtained and shown in Figure 9. There is an excellent agreement between the MKS and FEM predictions, attesting the scalability of the influence functions established in MKS framework. It should be noted that the computational efficiency is much more impressive with larger size microstructures. FEM simulation of $45 \times 45 \times 45$ microstructure took 3 minutes with 16 processors (each $3.0 \mathrm{GHz}$ ) on a supercomputer, while MKS prediction took only 24 seconds with only 1 processor $(3.0 \mathrm{GHz})$ on a standard computer.

\section{[Insert Figure 9 here]}

In this new MKS framework for a family of single phase cubic polycrystalline aggregates, it is once again observed that the first set of terms are adequate in predicting the response field of 
interest for low to moderate contrast material systems. However from our previous experiences [22], it is crucial that the higher order terms are included in the local state description for higher anisotropy levels (produces higher contrast). Especially in applications where higher accuracy in prediction of response field is required on the spatial locations where failure is likely to occur (high cycle fatigue, etc.), the higher order terms along with much more sophisticated error measures will be required.

\section{Conclusions}

In this paper, a generalized MKS framework has been presented to demonstrate the versatility and computational advantages of casting localization linkages in suitable Fourier representations, especially for capturing high dimensional local state spaces of interest encountered in advanced material systems. A general framework was presented to address this challenge and its applicability was demonstrated through a case study that involved the prediction of elastic strain fields in a broad class of cubic polycrystalline microstructures. The case study demonstrated the tremendous computational advantages of the new enhanced MKS framework presented in this paper. In particular, it was shown that spectral representations of the influence functions provide highly compact representations. The new generalized MKS framework will open new avenues for accomplishing efficient scale-bridging in hierarchical multiscale modeling and simulation of a wide range of material systems and physical phenomena.

\section{Acknowledgements}

Authors acknowledge funding from the Office of Naval Research (ONR) award N00014-11-10759 (Dr. William M. Mullins, program manager). 


\section{References}

[1] Kröner E. Statistical Modelling. In: Gittus J, Zarka J, editors. Modelling Small Deformations of Polycrystals. Springer Netherlands, 1986. p.229.

[2] Kröner E. J Mech Phy Solids 1977;25:137.

[3] Adams BL, Olson T. Prog Mater Sci 1998;43:1.

[4] Beran M, Mason T, Adams B, Olsen T. J Mech Phy Solids 1996;44:1543.

[5] Milton GW. The theory of composites: Cambridge University Press, 2002.

[6] Beran MJ, Pytte A. American Journal of Physics 1968;36:923.

[7] Michel J, Moulinec H, Suquet P. Computer methods in applied mechanics and engineering 1999;172:109.

[8] Moulinec H, Suquet P. Computer methods in applied mechanics and engineering 1998;157:69.

[9] Adams BL, Kalidindi SR, Fullwood DT. Microstructure Sensitive Design for Performance Optimization: Elsevier Science, 2012.

[10] Torquato S. Random heterogeneous materials: microstructure and macroscopic properties: Springer, 2002.

[11] Lin S, Garmestani H. Composites Part B: Engineering 2000;31:39.

[12] Garmestani H, Lin S, Adams B, Ahzi S. J Mech Phy Solids 2001;49:589.

[13] Mason TA, Adams BL. Metallurgical and Materials transactions A 1999;30:969.

[14] Adams BL, Gao X, Kalidindi SR. Acta Mater 2005;53:3563.

[15] Duvvuru HK, Wu X, Kalidindi SR. Comput Mater Sci 2007;41:138.

[16] Kalidindi SR, Landi G, Fullwood DT. Acta Mater 2008;56:3843.

[17] Kalidindi SR, Binci M, Fullwood D, Adams BL. Acta Mater 2006;54:3117. 
[18] Kalidindi SR, Niezgoda SR, Landi G, Vachhani S, Fast T. Comput Mater Continua 2010;17:103.

[19] Landi G, Kalidindi SR. Comput Mater Continua 2010;16:273.

[20] Landi G, Niezgoda SR, Kalidindi SR. Acta Mater 2010;58:2716.

[21] Fast T, Niezgoda SR, Kalidindi SR. Acta Mater 2011;59:699.

[22] Fast T, Kalidindi SR. Acta Mater 2011;59:4595.

[23] Kalidindi SR. ISRN Mater Sci 2012;2012:13.

[24] Yabansu YC, Patel DK, Kalidindi SR. Acta Mater 2014;81:151.

[25] Fullwood DT, Niezgoda SR, Adams BL, Kalidindi SR. Prog Mater Sci 2010;55:477.

[26] Proust G, Kalidindi SR. J Mech Phy Solids 2006;54:1744.

[27] Knezevic M, Kalidindi SR, Mishra RK. Int J Plast 2008;24:327.

[28] Knezevic M, Kalidindi SR. Comput Mater Sci 2007;39:643.

[29] Houskamp JR, Proust G, Kalidindi SR. Int J Multiscale Comput Eng 2007;5:261.

[30] Kalidindi SR, Houskamp JR, Lyons M, Adams BL. Int J Plast 2004;20:1561.

[31] Buehler MJ, Hartmaier A, Gao H. Modell Simul Mater Sci Eng 2004;12:S391.

[32] Groh S, Marin EB, Horstemeyer MF, Zbib HM. Int J Plast 2009;25:1456.

[33] Luscher DJ, McDowell DL, Bronkhorst CA. Int J Plast 2010;26:1248.

[34] Oden JT, Vemaganti K, Moës N. Computer Methods in Applied Mechanics and Engineering 1999;172:3.

[35] McDowell DL. Int J Plast 2010;26:1280.

[36] Olson GB. Science 1997;277:1237.

[37] Panchal JH, Kalidindi SR, McDowell DL. Comput Aided Design 2013;45:4.

[38] Shenoy M, Tjiptowidjojo Y, McDowell D. Int J Plast 2008;24:1694. 
[39] Al-Harbi HF, Landi G, Kalidindi SR. Modell Simul Mater Sci Eng 2012;20:055001.

[40] Bunge HJ. Texture analysis in materials science: mathematical methods: Butterworths, 1982.

[41] Wheeler D, Brough D, Fast T, Kalidindi SR, Reid A. PyMKS: Materials Knowledge System in Python. vol. 2014, 2014.

[42] Patel DK, Al-Harbi HF, Kalidindi SR. Acta Mater 2014;79:108.

[43] Adams BL, Henrie A, Henrie B, Lyon M, Kalidindi SR, Garmestani H. J Mech Phy Solids 2001;49:1639.

[44] Niezgoda SR, Yabansu YC, Kalidindi SR. Acta Mater 2011;59:6387.

[45] Niezgoda S, Kanjarla A, Kalidindi S. Integr Mater Manuf Innov 2013;2:1.

[46] Hibbit, Karlsson, Sorensen. ABAQUS/Standard Analysis User's Manual: Hibbit, Karlsson, Sorensen Inc., 2007.

[47] Proust G, Kalidindi SR. J Mech Phy Solids 2006;54:1744. 


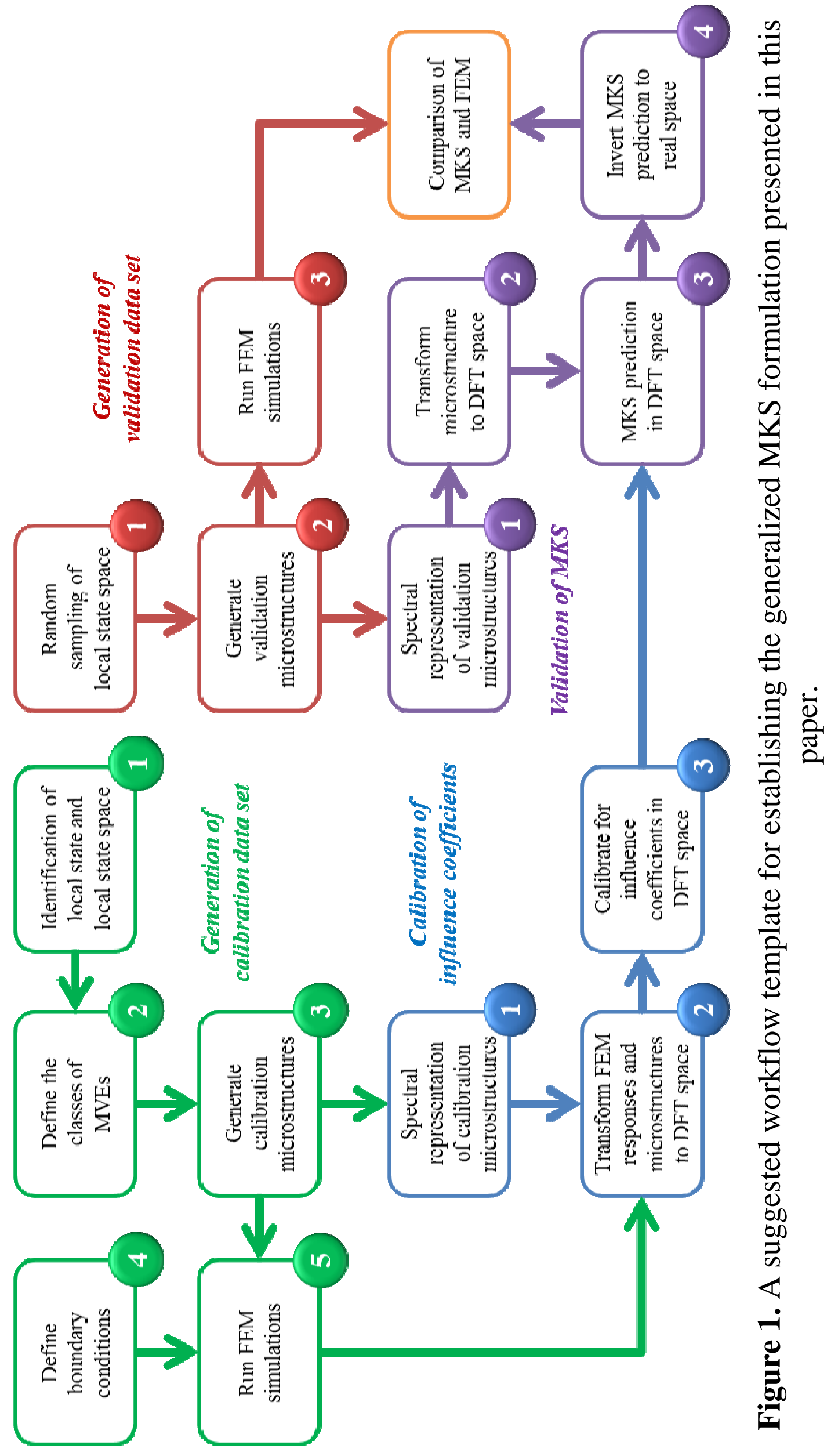



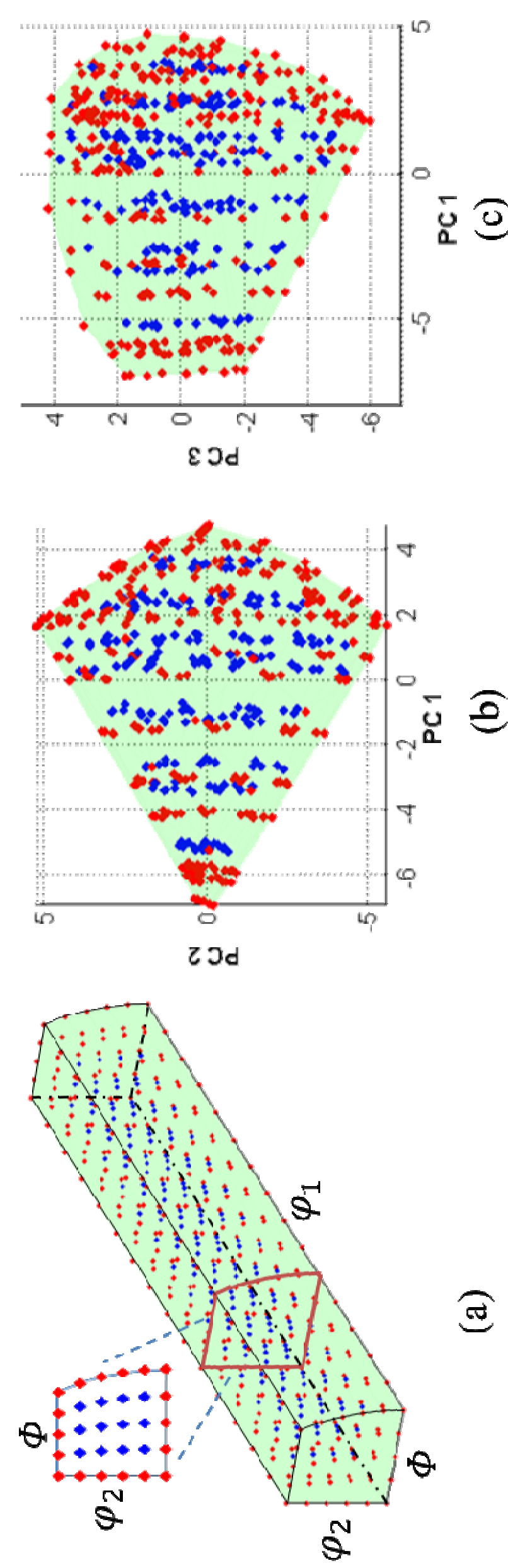

ช

บ

I 0

400

$-200 \cdot$

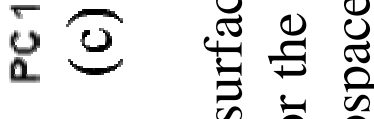

\&

วิ은

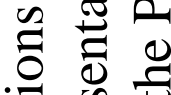

ช \&

를을

。

U

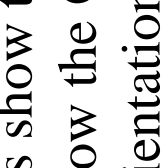

告 号

워 $\frac{\sigma}{\pi}$

ঠृ

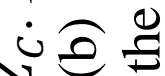

i $\dot{0}$

仓ี

홍

응

ฮี

当 气

. ․ㅡㄹ

를

ฮ

을

只

ชิ

ㅅ․ㄹ응

$\Perp 8$

bo 0

央党 


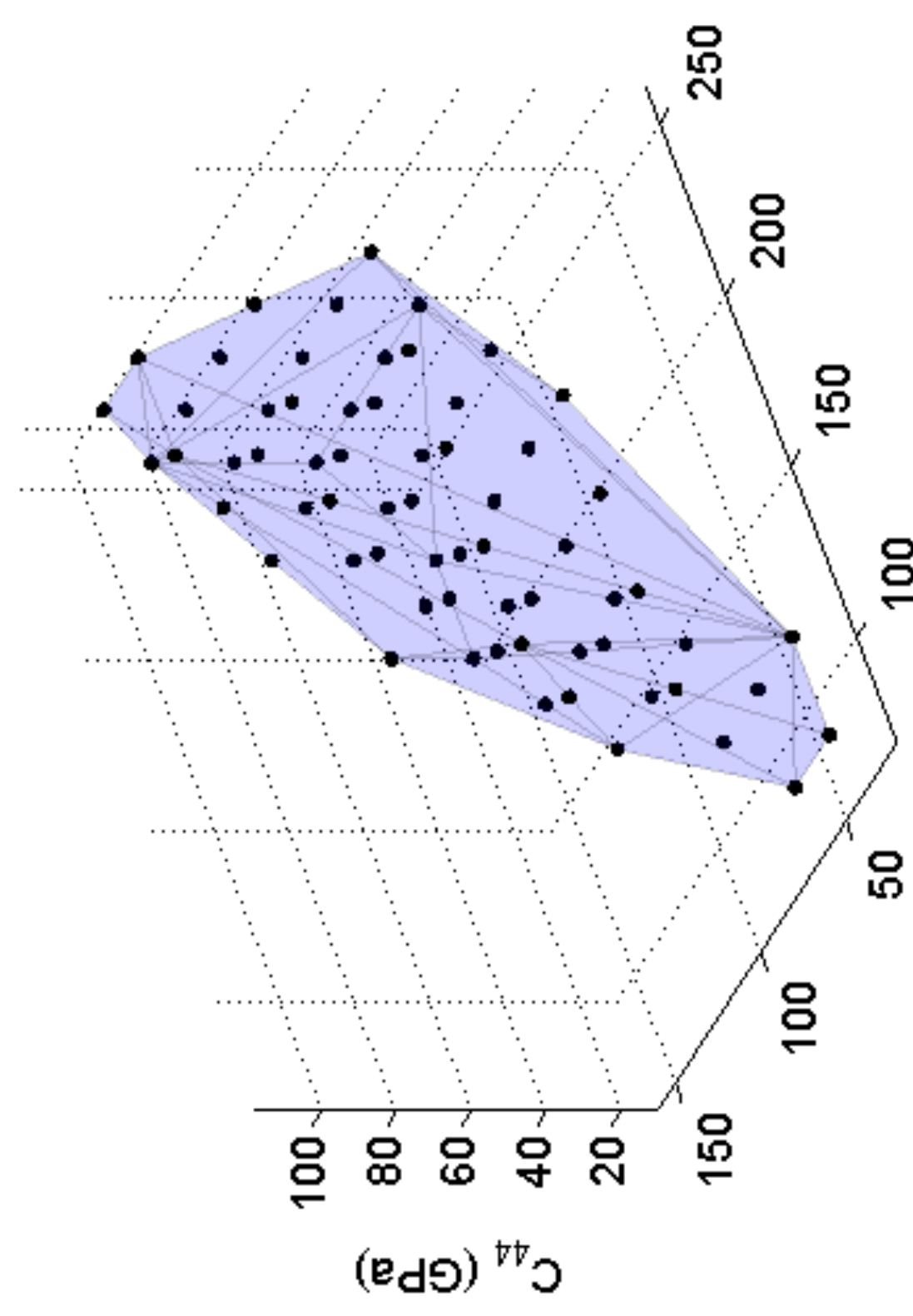

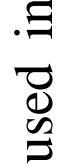

म্ं

जे

ثें

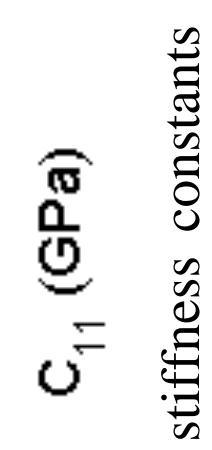

응 $\frac{0}{\frac{0}{2}}$

$\stackrel{8}{\Xi}$

4

กิ

오

ส

U

उ

प๐

屯

E

ம

บ

$\exists$

007 

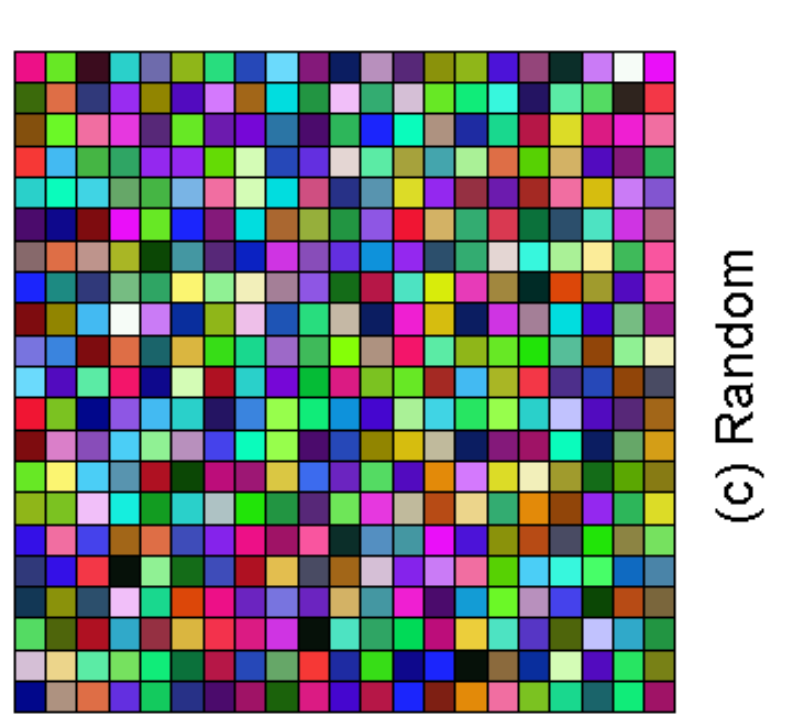

$\stackrel{9}{ \pm}$

$\exists \stackrel{0}{0}$

ชูป

$\frac{2}{0}$ 元

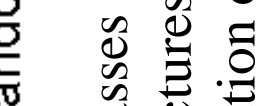

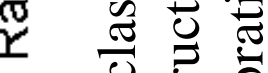

(U) I

$\geq$ U

$\sum \cdot$ 邑

\& ह.

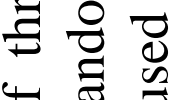

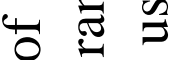

등 巳

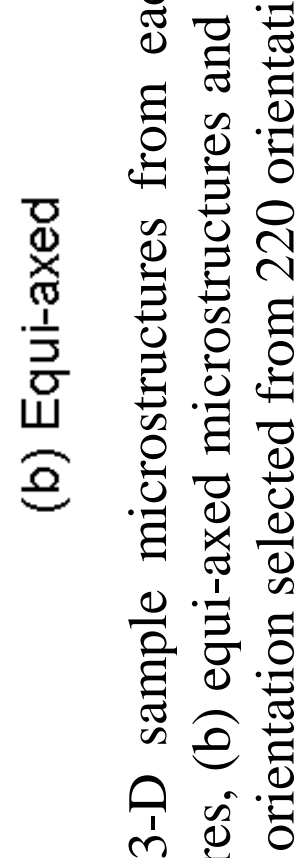
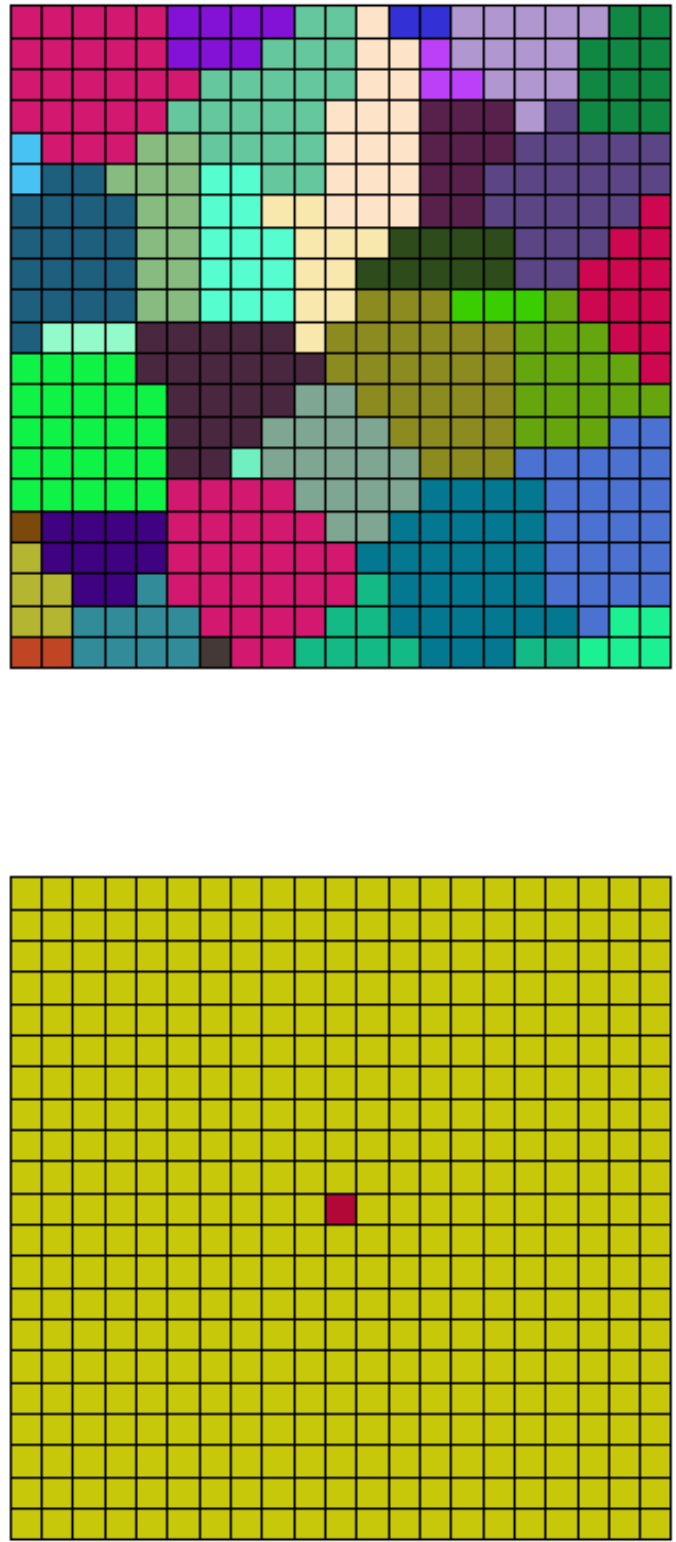

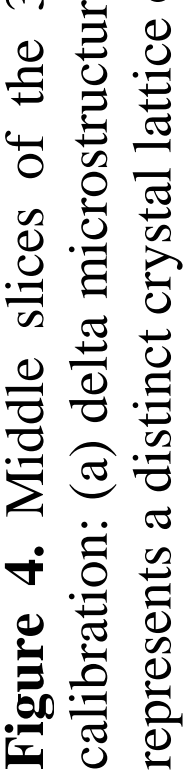


$\cong$

뭉

:

을

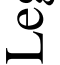

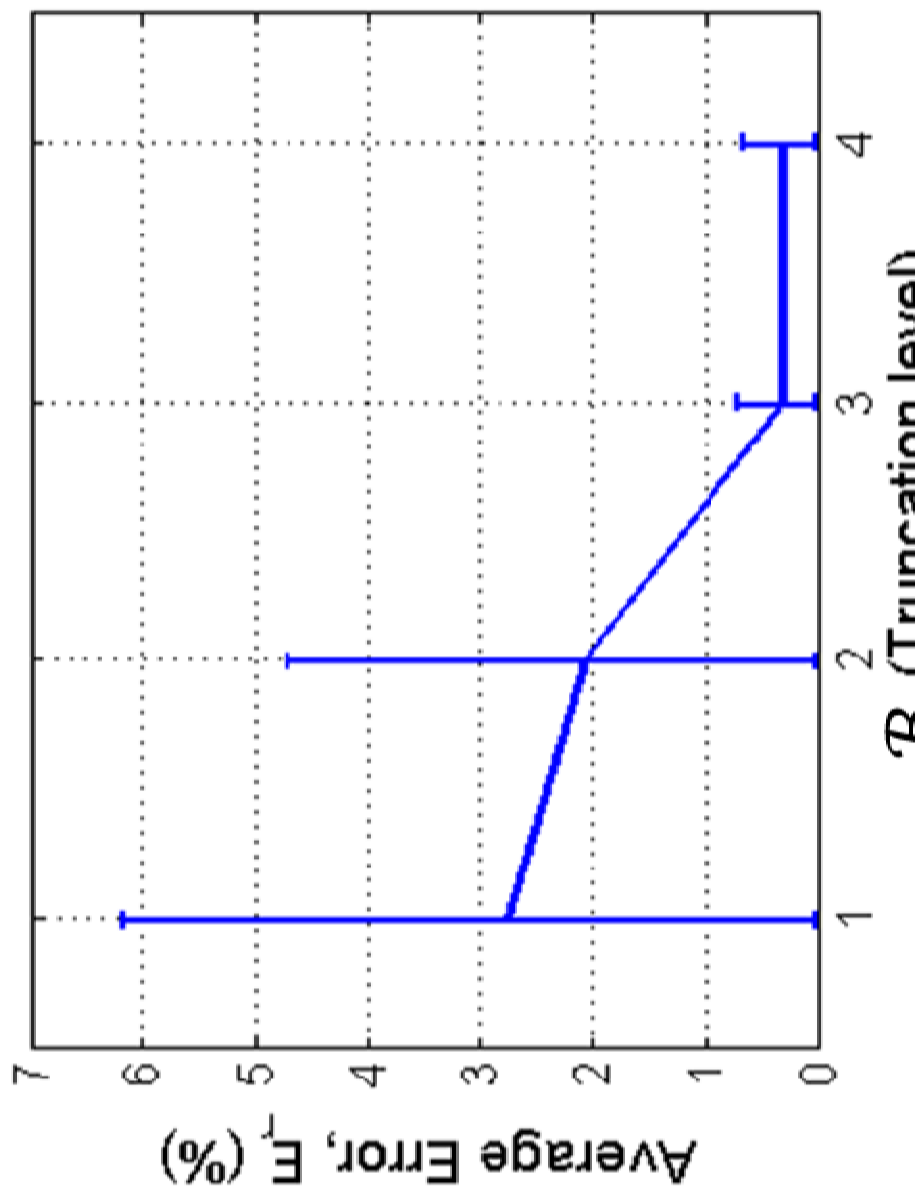

4

$\stackrel{\Xi}{2}$

จ

드응

Uै

อี

$\sigma$

สิ

ల్ర

के कि

이

当

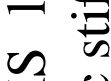

is

光

$\stackrel{0}{ \pm}$

. ฮั

을

디

i

氙 


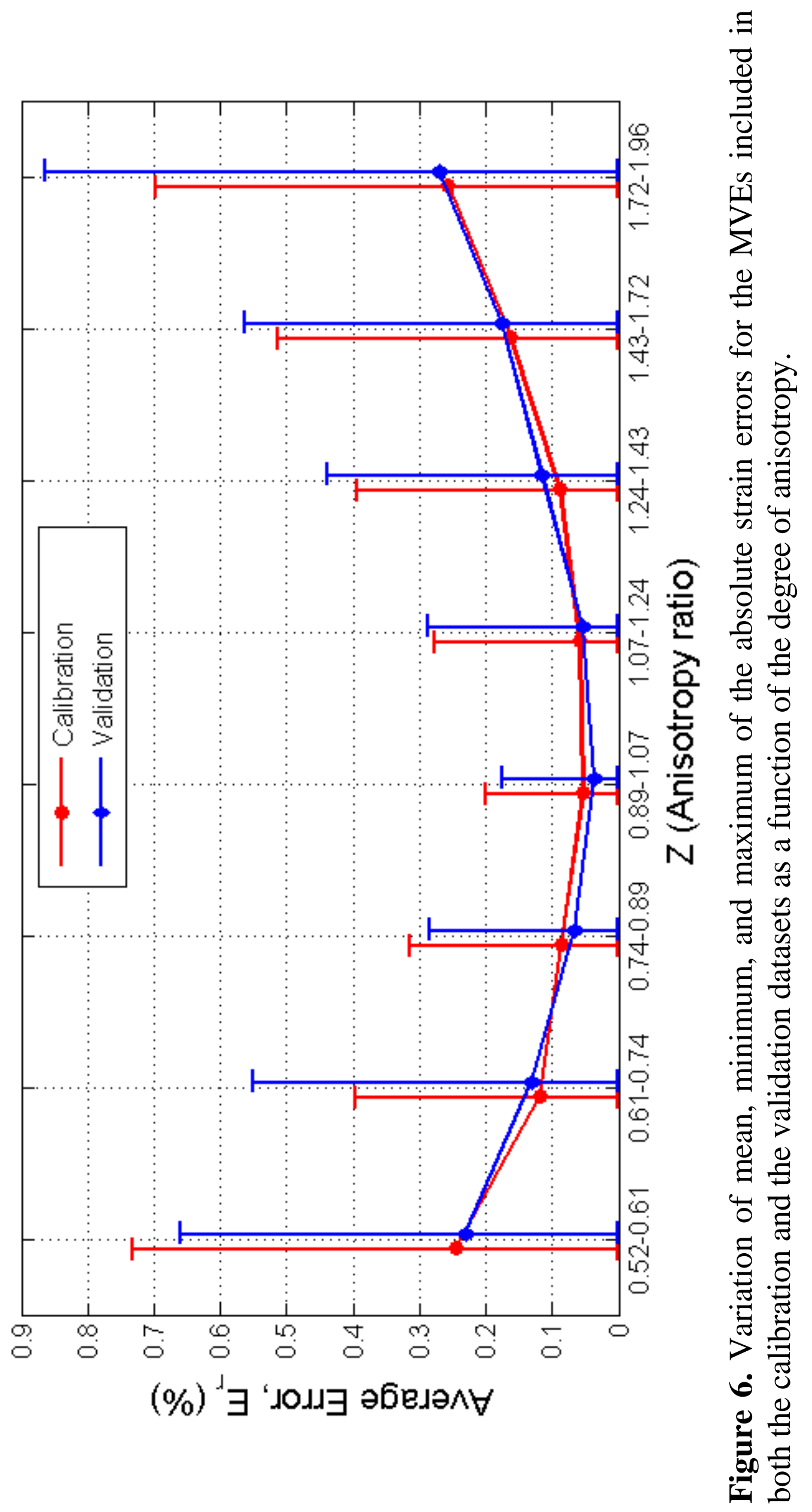



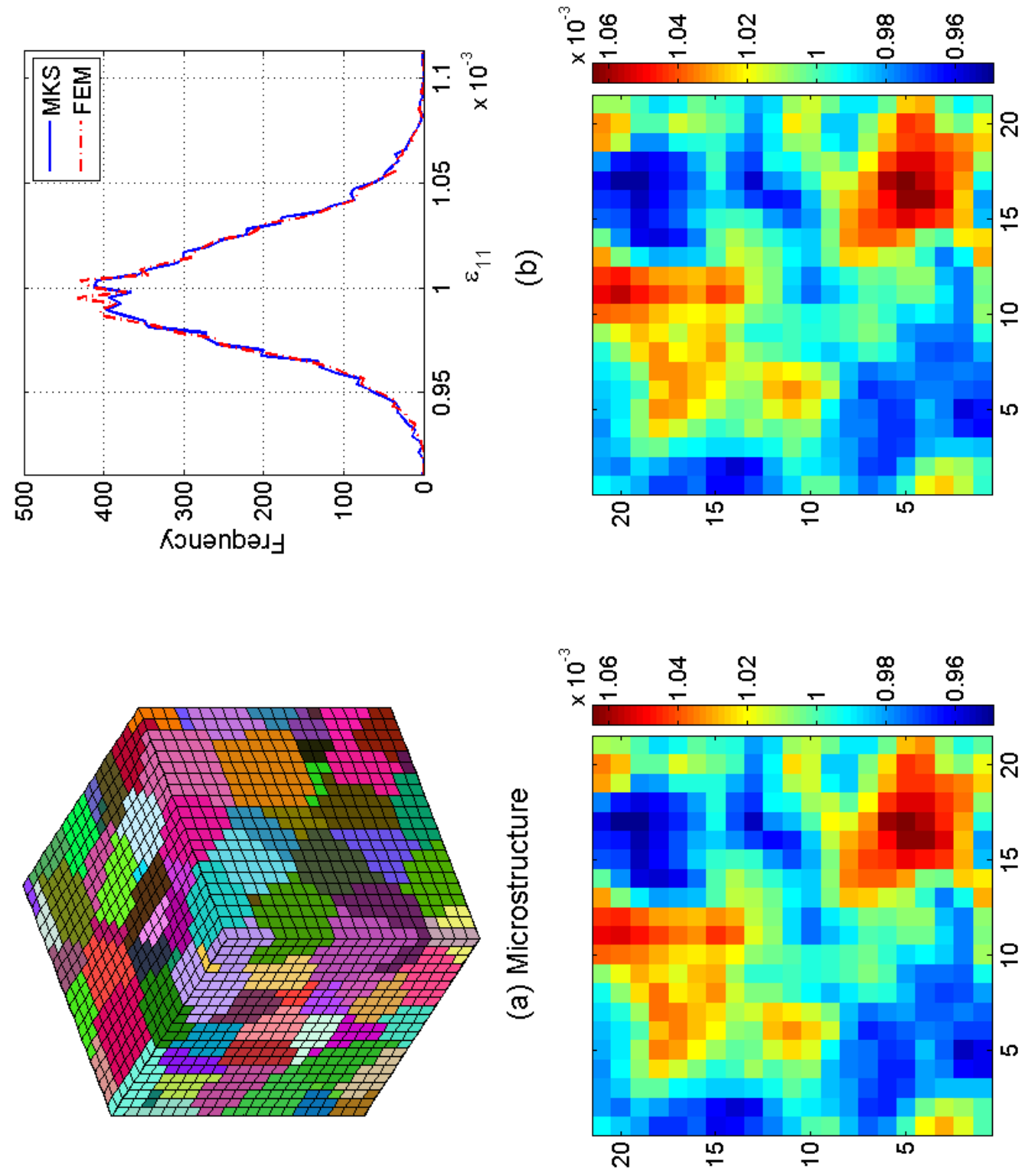

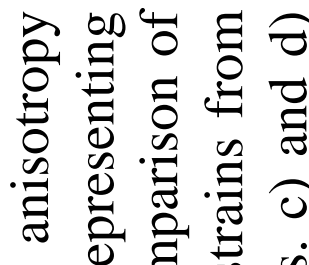
. 政

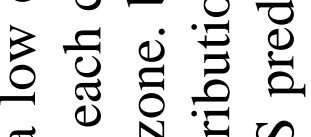

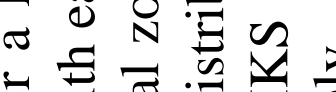

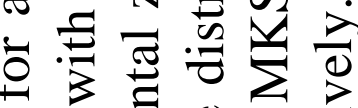
1

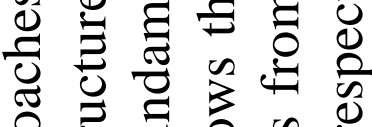

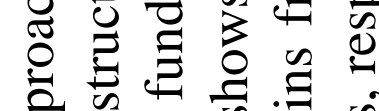
产 :

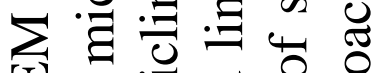

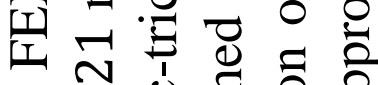

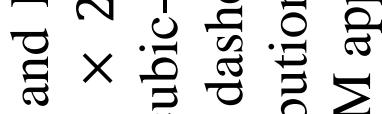

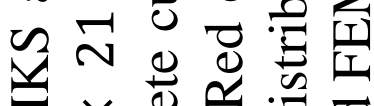
$\sum \times \frac{0}{2}: 0$

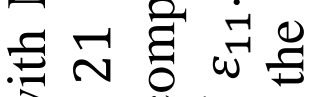

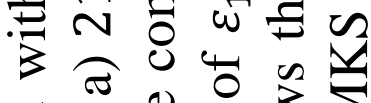

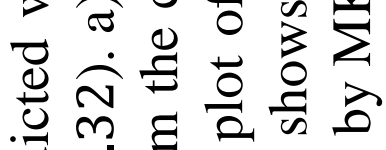

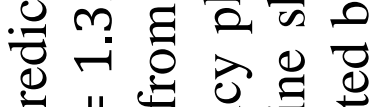
ㄴ. II

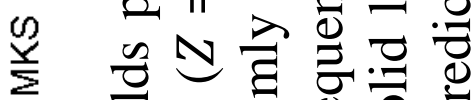
월

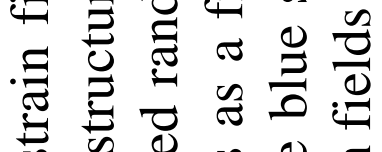
a पे 하의

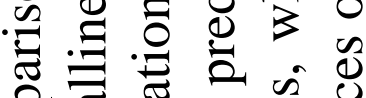

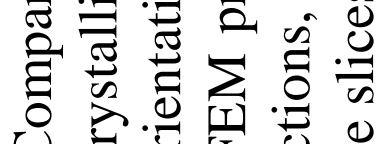

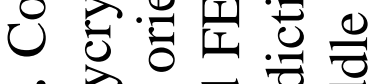

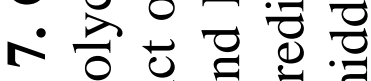

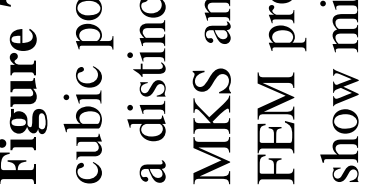




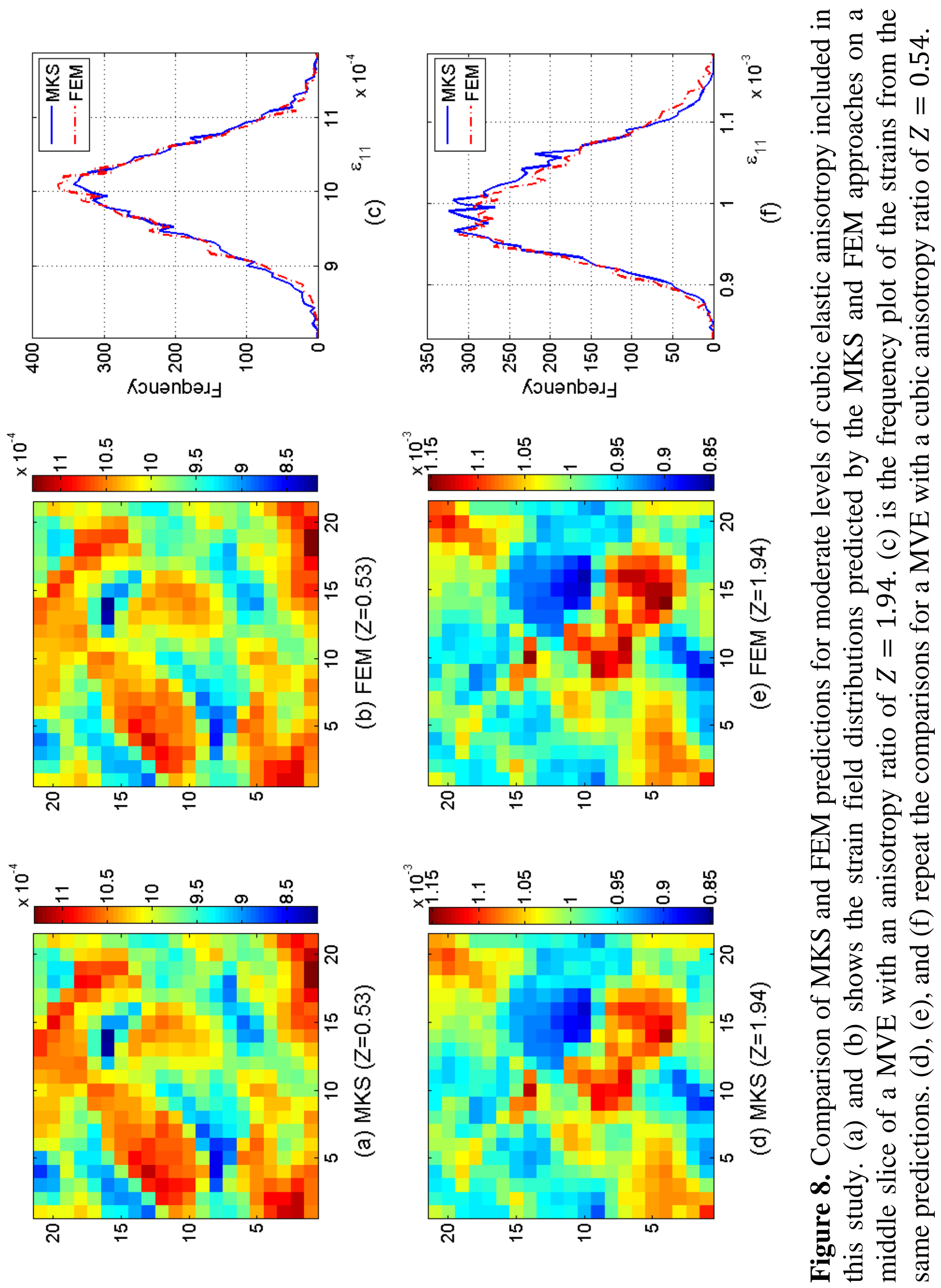




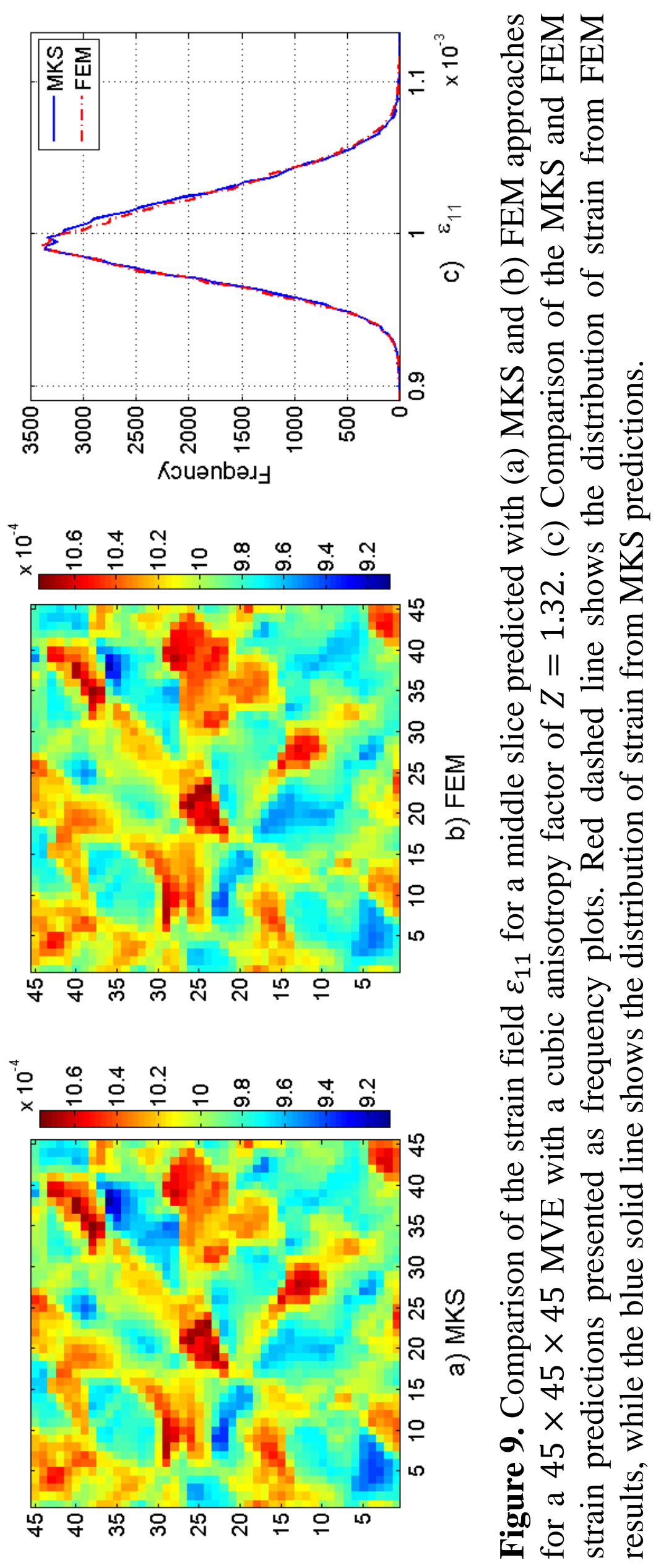

